\title{
Muon $g-2$ and coannihilating dark matter in the minimal supersymmetric standard model
}

\author{
Peter Cox $\odot,{ }^{1, *}$ Chengcheng Han, ${ }^{2, \dagger}$ and Tsutomu T. Yanagida ${ }^{3, \$}$ \\ ${ }^{1}$ School of Physics, The University of Melbourne, Victoria 3010, Australia \\ ${ }^{2}$ School of Physics, Sun Yat-Sen University, Guangzhou 510275, China \\ ${ }^{3}$ Tsung-Dao Lee Institute \& School of Physics and Astronomy, Shanghai Jiao Tong University, \\ 200240 Shanghai, China
}

(Received 26 April 2021; accepted 20 September 2021; published 27 October 2021)

\begin{abstract}
We demonstrate that the recent measurement of the anomalous magnetic moment of the muon and dark matter can be simultaneously explained within the minimal supersymmetric standard model. Dark matter is a mostly bino state, with the relic abundance obtained via coannihilations with either the sleptons or wino. The most interesting regions of parameter space will be tested by the next generation of dark matter direct detection experiments.
\end{abstract}

DOI: 10.1103/PhysRevD.104.075035

\section{INTRODUCTION}

The recent measurement of the anomalous magnetic moment of the muon [1] represents an exciting hint for the existence of physics beyond the Standard Model. The combination of the new result and the Brookhaven (E821) measurement [2] is in tension with the Standard Model prediction $^{1}[8]$ at $4.2 \sigma$ :

$a_{\mu}^{\exp }-a_{\mu}^{\mathrm{SM}}=\left(2.51 \pm 0.41_{(\exp )} \pm 0.43_{\text {(theory) }}\right) \times 10^{-9}$.

Supersymmetry is one of the leading candidates to explain this discrepancy. In this paper, and in light of the new measurement, we explore the possibility that the minimal supersymmetric standard model (MSSM) could be responsible for both the deviation in the muon $g-2$ and the dark matter $(\mathrm{DM})$ of the universe.

The MSSM features a natural dark matter candidate in the form of the lightest neutralino. It is by now well understood that the most promising scenario to simultaneously explain the deviation in $a_{\mu}$ and obtain the observed relic abundance via thermal production involves binolike dark matter and coannihilations [9-13]. This conclusion is

\footnotetext{
peter.cox@unimelb.edu.au

hanchch@mail.sysu.edu.cn

tsutomu.tyanagida@sjtu.edu.cn

${ }^{1}$ This uses the $R$-ratio method [3-6] for the HVP contribution. A recent lattice determination [7] yields a different value which, if correct, would alleviate the tension in Eq. (1).

Published by the American Physical Society under the terms of the Creative Commons Attribution 4.0 International license. Further distribution of this work must maintain attribution to the author(s) and the published article's title, journal citation, and DOI. Funded by SCOAP ${ }^{3}$.
}

primarily driven by the strong bounds from dark matter direct detection, combined with the fact that it is not possible to obtain a large enough contribution to $a_{\mu}$ for either pure Higgsino or wino dark matter.

There are two distinct scenarios to consider, depending on the identity of the coannihilating partner: bino-slepton and bino-wino coannihilation. As we shall demonstrate, both of these scenarios have regions of parameter space that can explain the result in Eq. (1) while simultaneously accounting for the dark matter relic abundance and evading all other constraints.

\section{ANALYSIS FRAMEWORK AND ASSUMPTIONS}

We begin by describing the details of our analysis. First, we assume that the squarks, gluinos and additional Higgs bosons are all decoupled, motivated by the strong bounds from collider searches. For concreteness we fix their masses to be $\sim 3 \mathrm{TeV}$. This is also the renormalization scale at which all the parameters are specified. The trilinear coupling $A_{t}$ is fixed to $5 \mathrm{TeV}$ in order to obtain a Higgs mass of $\approx 125 \mathrm{GeV}$, and we assume that all other $A$-terms are negligible.

To simplify our analysis, we assume that the left and right-handed slepton soft masses are equal. We also take equal slepton soft masses for the first and second generations. We focus on the case where $\operatorname{sgn}\left(M_{1,2} \mu\right)>0$, since this ensures that the dominant contributions to $a_{\mu}$ have the correct sign to account for the difference in Eq. (1).

We use the spectrum generator SuSpect-2.52 [14], while the dark matter relic abundance, DM-nucleon scattering cross-section, and one-loop SUSY contributions to $a_{\mu}$ are all calculated using MicrOMEGAs - 5.2.7 [15]. We also include the leading two-loop contributions to $a_{\mu}$. These come from $\tan \beta$-enhanced corrections to the muon 
Yukawa coupling $[16,17]$ and the QED running down to the muon mass scale [18].

\section{BINO-STAU COANNIHILATION}

The first scenario we consider is bino-slepton coannihilation with universal slepton masses. The assumption of universal slepton masses is often imposed in order to avoid dangerous contributions to flavor changing neutral current (FCNC) processes. It can be motivated by certain supersymmetry breaking scenarios, such as gaugino mediation [19-21]. In this case, the NLSP and coannihilating partner is the lightest stau. Achieving the correct relic abundance then requires a mass-splitting $m_{\tilde{\tau}_{1}}-m_{\chi_{1}^{0}} \lesssim$ $15 \mathrm{GeV}$.

The stau coannihilation region is shown in Fig. 1, where we have taken $M_{2}=1 \mathrm{TeV} . M_{1}$ is adjusted across the parameter space in order to obtain the correct relic abundance. Consider first the left panel where we fix $\tan \beta=50$. The green (blue) regions fit the $a_{\mu}$ measurement at $1 \sigma(2 \sigma)$. However, the $1 \sigma$ region is already excluded by LHC searches for the first and second generation sleptons [22,23] (red line).

There is also a constraint from vacuum stability, due to the existence of charge-breaking minima in the scalar potential when $\mu \tan \beta$ becomes large [26]. We take the bound from Ref. [27]; the grey region is excluded at zero temperature, while a stronger bound (black line) is obtained by considering the finite temperature effective potential and requiring stability throughout the thermal history of the universe (note that this assumes a sufficiently high reheating temperature).

The right panel of Fig. 1 corresponds to $\tan \beta=20$. The smaller value of $\tan \beta$ has the effect of compressing the slepton spectrum, which relaxes the bounds from LHC slepton searches. There are then regions which can fit $a_{\mu}$ at $1 \sigma$, while evading the bounds from collider searches. These correspond to very small slepton masses, with the lightest stau close to the LEP lower bound.

It is clear from fig. 1 that bino-stau coannihilation with universal slepton masses, while still viable, is strongly constrained. One could consider decreasing $M_{2}$ in order to increase the chargino-sneutrino contribution to $a_{\mu}$. This would move the best-fit region toward larger slepton masses and away from the collider bounds. However, $M_{2}$ cannot be decreased significantly without encountering bounds from chargino searches, particularly from sleptonmediated decays [22].

While LHC searches currently provide the strongest constraints on this scenario, dark matter direct detection will have an important role in the future. The entirety of the best-fit regions in both panels of Fig. 1 will be probed by the LZ experiment [28]. Indirect detection is not currently sensitive to any of the coannihilation scenarios, due to the small annihilation cross section today. In the future, the Cherenkov Telescope Array should have sensitivity to the bino-stau coannihilation scenario [29].

Finally, note that we are assuming universal slepton soft masses at low-scale. In a UV model this relation might be expected to hold at high scales, but the RG running tends to reduce the stau soft mass compared to the first and second generation sleptons. This may lead to slightly stronger bounds from slepton searches for small $\tan \beta$, but we do not expect this effect to significantly alter our conclusions (see also [30]).
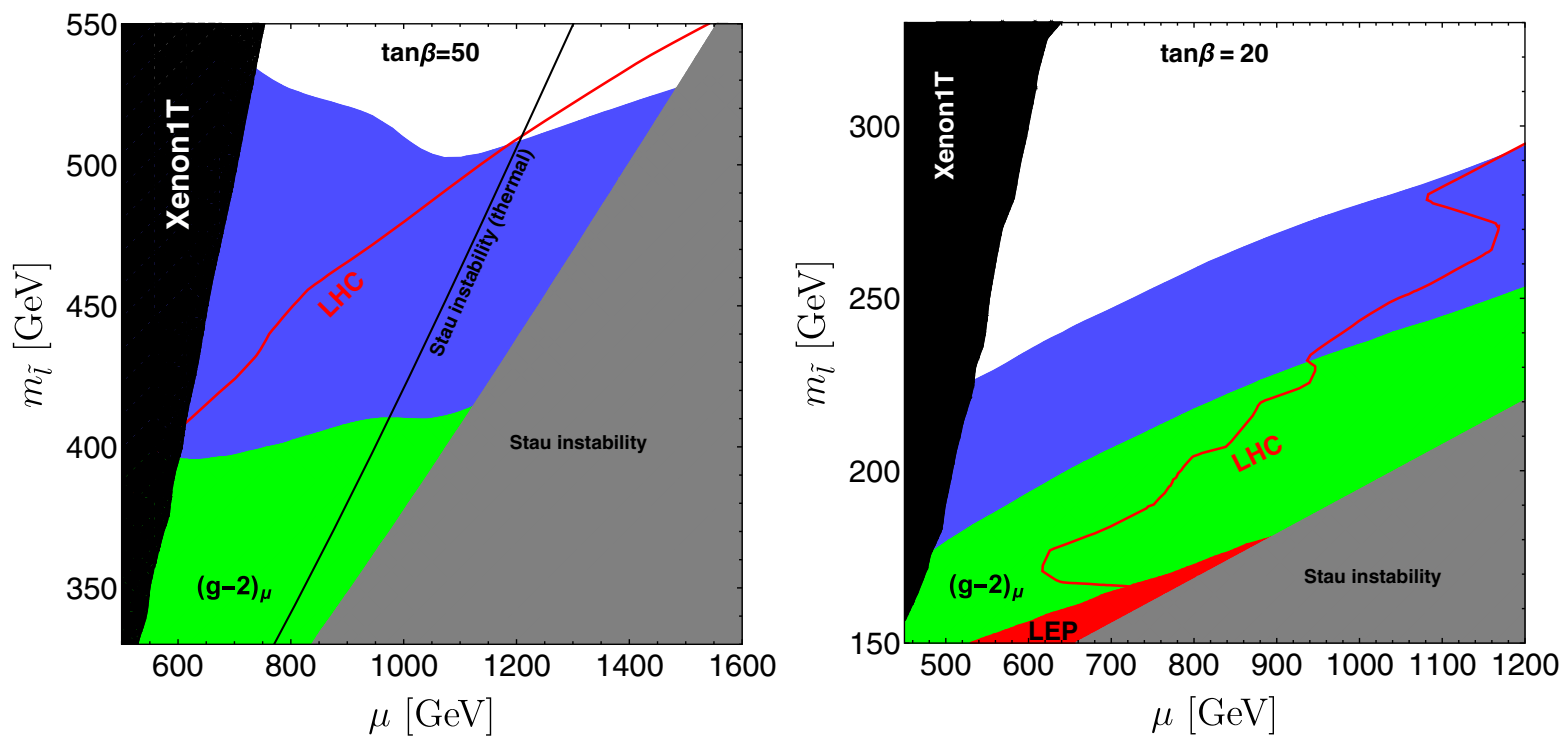

FIG. 1. Bino-stau coannihilation. $M_{1}$ has been adjusted to obtain the correct relic abundance, and we fix $M_{2}=1 \mathrm{TeV}$. The green (blue) region is consistent with $a_{\mu}$ at $1 \sigma(2 \sigma)$. The black region is excluded by XENON1T [24]. The region to the right of the red line is excluded by the ATLAS slepton search with $139 \mathrm{fb}^{-1}$ [22], and the red region is excluded by slepton searches at LEP [25]. The grey region is excluded by vacuum instability. Left: $\tan \beta=50$. Right: $\tan \beta=20$. 

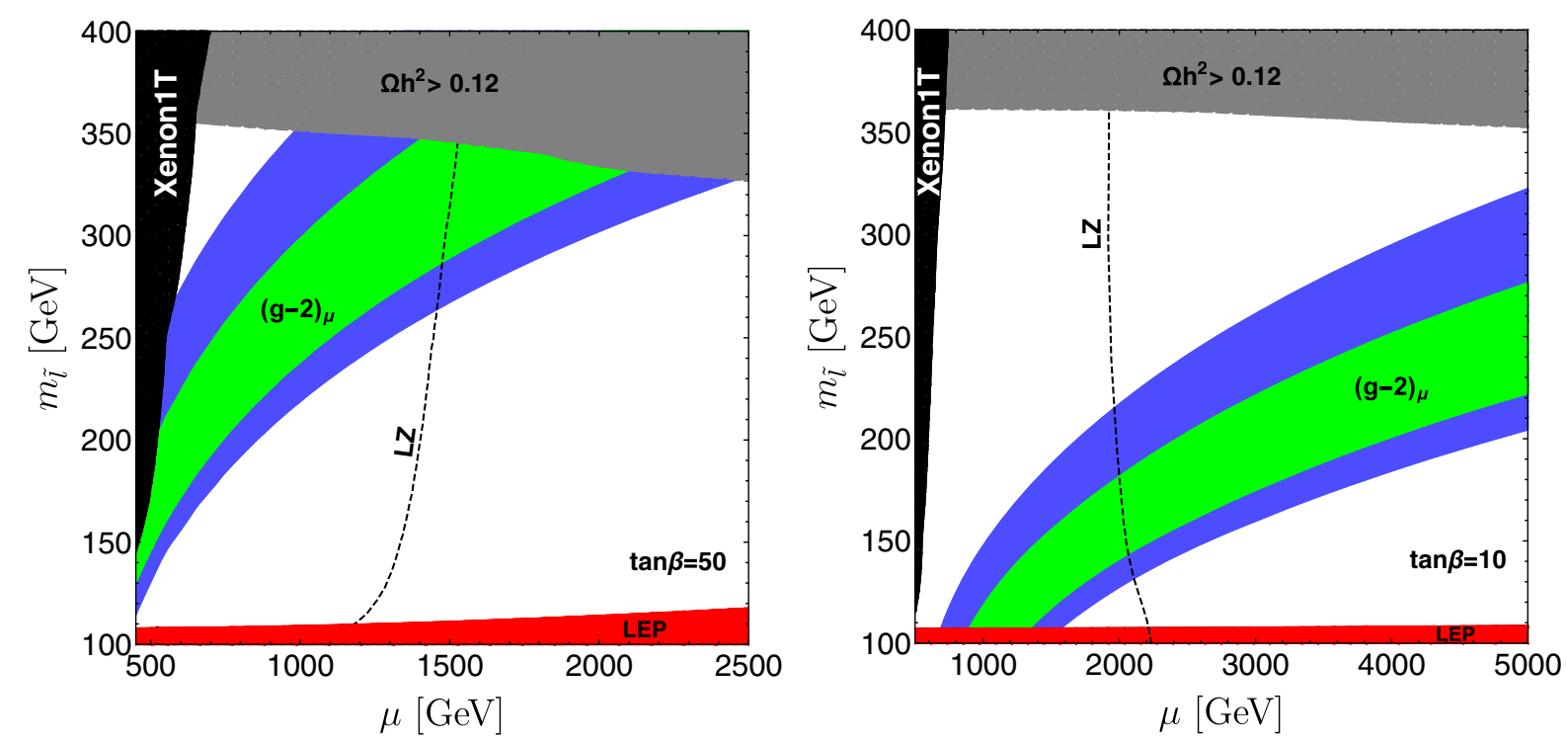

FIG. 2. Bino-slepton coannihilation. We have fixed $\tan \beta=50$ (left) and $\tan \beta=10$ (right). $M_{1}$ has been adjusted to obtain the correct relic abundance; for large slepton masses this becomes impossible as shown by the grey region. The green (blue) region is consistent with $a_{\mu}$ at $1 \sigma(2 \sigma)$. The black region is excluded by XENON1T [24], while the dashed line shows the future sensitivity of the LZ experiment [28]. The red shaded region is excluded by slepton searches at LEP [25].

\section{BINO-SLEPTON COANNIHILATION}

Given the results of the previous section, we now relax the assumption of flavor universality for the slepton masses and assume that the staus are decoupled $\left(m_{\tilde{\tau}_{L}}=\right.$ $m_{\tilde{\tau}_{R}}=3 \mathrm{TeV}$ ). As we shall see, this opens up significant regions of parameter space in which both the muon $g-2$ and dark matter can be accommodated. This type of spectrum can be realized, for example, in Gaugino + Higgs mediation [30], ${ }^{2}$ without inducing large FCNCs [33].

In this scenario the NLSP is the lightest smuon. The results are shown in fig. 2 for $\tan \beta=50(10)$ in the left (right) panels. $M_{1}$ has been adjusted to obtain the observed relic abundance, which requires a masssplitting $m_{\tilde{\mu}_{1}}-m_{\tilde{\chi}_{1}^{0}} \lesssim 20 \mathrm{GeV}$. The required mass-splitting decreases for larger slepton masses, and there is an upper bound on the slepton mass (grey region) above which it is no longer possible to obtain the correct relic density. When $\mu \tan \beta$ is small, the lightest selectron and smuon are almost degenerate and both act as coannihilation partners. As $\mu \tan \beta$ is increased, $\tilde{\mu_{1}}$ becomes lighter due to the left-right mixing and plays the dominant role in setting the relic abundance. This also leads to a decrease in the upper bound on the slepton mass.

Here, we have assumed that the wino is decoupled $\left(M_{2}=3 \mathrm{TeV}\right)$; the dominant contribution to $a_{\mu}$ therefore comes from the bino-smuon loop. This contribution is proportional to the left-right smuon mixing and is enhanced for large $\mu \tan \beta$. Hence, the best-fit region for $a_{\mu}$ moves

\footnotetext{
${ }^{2}$ This is achieved with large negative soft masses for the Higgs doublets at the GUT scale, and $\mu \sim \mathcal{O}(10) \mathrm{TeV}[31,32]$.
}

toward higher slepton masses as $\mu$ is increased. For large $\tan \beta$, much of the best-fit region will be probed in the relatively near future by dark matter direct detection. Reducing $\tan \beta$ has the effect of moving the best-fit region for $a_{\mu}$ to lower slepton masses for a given $\mu$. This is seen in the right panel of Fig. 2, where there is significant viable parameter space well beyond the reach of future direct detection experiments.

We now briefly discuss collider searches for the light sleptons. The compressed spectrum makes this scenario challenging to probe at hadron colliders. Nevertheless, there is a dedicated ATLAS search targeting bino-slepton coannihilation [34]. However, it does not currently constrain the parameter space in Fig. 2. Given the upper bound on the slepton mass from the relic abundance, the binoslepton coannihilation scenario could, however, be fully tested at a future lepton collider with $\sqrt{s}>700 \mathrm{GeV}$ (see Refs. [35,36]).

\section{BINO-WINO COANNIHILATION}

Last, we consider bino-wino coannihilation. In this scenario a slightly larger mass-splitting of $10-30 \mathrm{GeV}$ is needed between the binolike LSP and winolike NLSP [37,38]. This situation is shown in Fig. 3 for $M_{2}=400 \mathrm{GeV}$ and $\tan \beta=50$. We have fixed $M_{2}-M_{1}=28 \mathrm{GeV}$, which gives approximately the correct relic abundance across the parameter space (the precise mass-splitting needed has a mild $\mu$-dependence). There are significant regions of parameter space that can explain both $a_{\mu}$ and the dark matter abundance. For large $\mu$, the binosmuon contribution to $a_{\mu}$ dominates, while for $\mu \lesssim 1.5 \mathrm{TeV}$ 


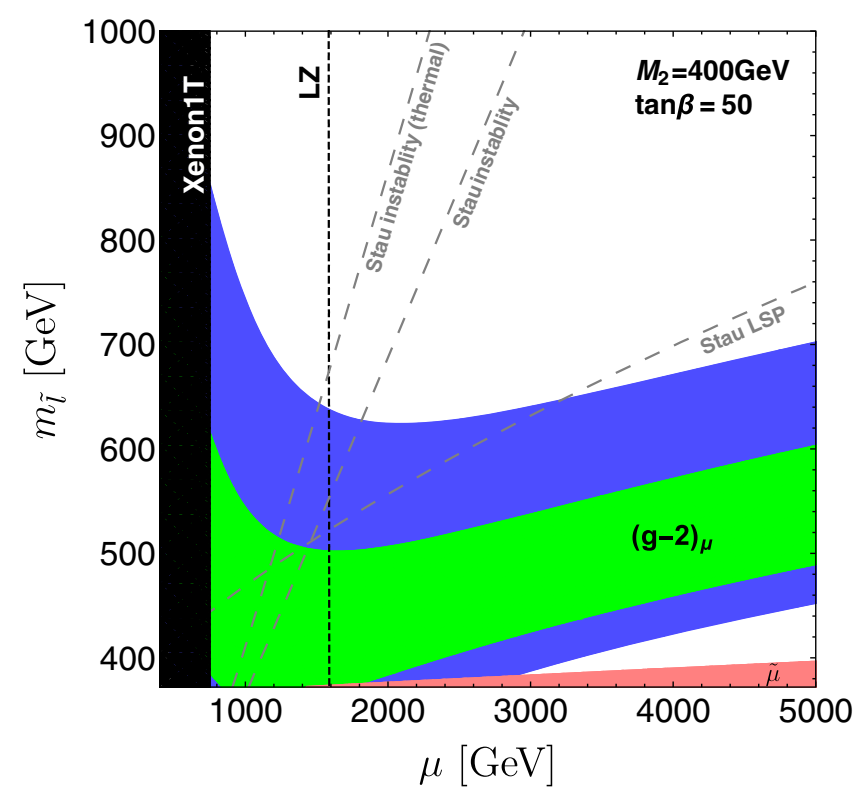

FIG. 3. Bino-wino coannihilation. We have fixed $\tan \beta=50$ and $M_{2}=400 \mathrm{GeV}$, with $M_{2}-M_{1}=28 \mathrm{GeV}$ to obtain the correct relic abundance. The LSP mass is $m_{\tilde{\chi}_{1}^{0}} \approx 370 \mathrm{GeV}$. The green (blue) region is consistent with $a_{\mu}$ at $1 \sigma(2 \sigma)$. The black region is excluded by XENON1T [24], while the black dashed line shows the future sensitivity of the LZ experiment [28]. In the pink region $\tilde{\mu}_{1}$ becomes the LSP. The grey dashed lines apply only for universal slepton masses, in which case the region to the right is excluded.

the chargino-sneutrino contribution becomes important and moves the $g-2$ best-fit region to higher slepton masses. This latter region will be probed by future direct detection experiments.

Collider searches for the sleptons and wino can potentially provide powerful probes of this scenario, although do not currently constrain the parameter space shown in Fig. 3. Both ATLAS [34] and CMS [39] have dedicated chargino searches targeting the compressed spectra relevant for bino-wino coannihilation. Currently, the CMS search with $137 \mathrm{fb}^{-1}$ obtains a bound of $m_{\tilde{\chi}_{2}^{0}} / \tilde{\chi}_{ \pm}^{1}>280 \mathrm{GeV}$ for $\Delta m=10 \mathrm{GeV}$, reducing to $m_{\tilde{\chi}_{2}^{0} / \tilde{\chi}_{ \pm}^{1}}>200 \mathrm{GeV}$ for $\Delta m=30 \mathrm{GeV}$. These limits assume that $\tilde{\chi}_{2}^{0}, \tilde{\chi}_{ \pm}^{1}$ decay purely via off-shell gauge bosons. Here, there can also be decays to leptons mediated via the light sleptons, which strengthens the bounds slightly. At the HL-LHC these searches are projected to eventually be sensitive to $m_{\tilde{\chi}_{2}^{0} / \tilde{\chi}_{ \pm}^{1}} \lesssim 430 \mathrm{GeV}[40]$.

Slepton searches can already probe masses up to $700 \mathrm{GeV}[22,41]$, although the limits become weaker with increasing LSP mass and there is currently no bound for $m_{\chi_{1}^{0}}>400 \mathrm{GeV}$. For smaller values of $M_{2}$ than shown in Fig. 3, slepton searches do constrain the parameter space. However, care should be taken when imposing the limits. First, the naive limit is weakened by the fact that the $\operatorname{BR}\left(\tilde{\ell}^{ \pm} \rightarrow \chi_{1,2}^{0} \ell^{ \pm}\right) \approx 60 \%$, since the decay mode $\tilde{\ell}^{ \pm} \rightarrow$ $\chi_{1}^{ \pm} \nu$ is also accessible. Second, in addition to slepton pair production, the processes $p p \rightarrow \tilde{\nu} \tilde{\ell}$ and $p p \rightarrow \tilde{\nu} \tilde{\nu}$, with $\tilde{\nu} \rightarrow \chi_{1}^{ \pm} \ell^{\mp}$, may also pass the analysis cuts. This is because the additional soft leptons or jets from the subsequent chargino decay may not be reconstructed. This has the potential to strengthen the limit due to the large $\tilde{\nu} \tilde{\ell}$ production cross section, but requires a full recasting of the analysis.

In the future, slepton searches at the (HL-)LHC are expected to probe most of the $2 \sigma$ region for $a_{\mu}$, with two exceptions. The first is the fully compressed region where $M_{1} \approx M_{2} \approx m_{\tilde{\ell}}$. The second is when $\mu$ becomes extremely large, in which case $a_{\mu}$ can be explained with smuon masses exceeding $1 \mathrm{TeV}$ [42].

So far in this section we have assumed that the staus are decoupled. Let us now briefly comment on the case of universal slepton masses. In this case the large- $\mu$ region is excluded due to the bound from vacuum stability or because the lightest stau becomes the LSP (grey dashed lines). The small surviving region that can explain $a_{\mu}$ will soon be tested by direct detection, although for smaller values of $\tan \beta$ this region moves beyond the reach of LZ.

\section{CONCLUSION}

The discrepancy between the measurement of the anomalous magnetic moment of the muon and its Standard Model prediction now exceeds $4 \sigma$, providing a tantalising hint for physics beyond the Standard Model. In this letter, we have demonstrated that this result can easily be accommodated within the framework of the MSSM, with a binolike LSP simultaneously responsible for dark matter. The observed relic abundance is achieved through coannihilations with either the sleptons or a light wino.

We find that bino-stau coannihilation with universal slepton masses is now strongly constrained by LHC searches and will be thoroughly tested by the LZ experiment. On the other hand, with nonuniversal slepton masses the majority of the best-fit region for $a_{\mu}$ currently remains unconstrained for both the bino-slepton and bino-wino coannihilation scenarios.

In both scenarios, the regions with $\mu \lesssim 1.5 \mathrm{TeV}$ will be probed by the next generation of dark matter direct detection experiments in the near future. This is especially interesting, given that this region is also theoretically preferred by naturalness.

\section{ACKNOWLEDGMENTS}

The authors would like to thank Norimi Yokozaki for useful discussions. The work of P. C. was supported by the Australian Government through the Australian Research Council. C. H. is supported by the Guangzhou Basic and Applied Basic Research Foundation under Grant No. 202102020885, and the Sun Yat-Sen University Science Foundation. T. T. Y. is supported by China Grant for Talent Scientific Start-Up Project. 
[1] B. Abi et al. (Muon $g-2$ Collaboration), Measurement of the Positive Muon Anomalous Magnetic Moment to 0.46 ppm, Phys. Rev. Lett. 126, 141801 (2021).

[2] G. W. Bennett et al. (Muon g-2 Collaboration), Final report of the muon E821 anomalous magnetic moment measurement at BNL, Phys. Rev. D 73, 072003 (2006).

[3] G. Colangelo, M. Hoferichter, and P. Stoffer, Two-pion contribution to hadronic vacuum polarization, J. High Energy Phys. 02 (2019) 006.

[4] M. Hoferichter, B.-L. Hoid, and B. Kubis, Three-pion contribution to hadronic vacuum polarization, J. High Energy Phys. 08 (2019) 137.

[5] M. Davier, A. Hoecker, B. Malaescu, and Z. Zhang, A new evaluation of the hadronic vacuum polarisation contributions to the muon anomalous magnetic moment and to $\boldsymbol{\alpha}\left(\mathbf{m}_{\mathbf{Z}}^{\mathbf{2}}\right)$, Eur. Phys. J. C 80, 241 (2020); Erratum, Eur. Phys. J. C 80, 410 (2020).

[6] A. Keshavarzi, D. Nomura, and T. Teubner, $g-2$ of charged leptons, $\alpha\left(M_{Z}^{2}\right)$, and the hyperfine splitting of muonium, Phys. Rev. D 101, 014029 (2020).

[7] S. Borsanyi et al., Leading hadronic contribution to the muon magnetic moment from lattice QCD, Nature (London) 593, 51 (2021).

[8] T. Aoyama et al., The anomalous magnetic moment of the muon in the Standard Model, Phys. Rep. 887, 1 (2020).

[9] M. Endo, K. Hamaguchi, S. Iwamoto, and K. Yanagi, Probing minimal SUSY scenarios in the light of muon $g-2$ and dark matter, J. High Energy Phys. 06 (2017) 031.

[10] E. Bagnaschi et al., Likelihood analysis of the pMSSM11 in Light of LHC 13-TeV Data, Eur. Phys. J. C 78, 256 (2018).

[11] P. Cox, C. Han, and T. T. Yanagida, Muon $g-2$ and dark matter in the minimal supersymmetric standard model, Phys. Rev. D 98, 055015 (2018).

[12] M. Abdughani, K.-I. Hikasa, L. Wu, J. M. Yang, and J. Zhao, Testing electroweak SUSY for muon $g-2$ and dark matter at the LHC and beyond, J. High Energy Phys. 11 (2019) 095.

[13] M. Chakraborti, S. Heinemeyer, and I. Saha, Improved $(g-2)_{\mu}$ Measurements and Supersymmetry, Eur. Phys. J. C 80, 984 (2020).

[14] A. Djouadi, J.-L. Kneur, and G. Moultaka, SuSpect: A fortran code for the supersymmetric and Higgs particle spectrum in the MSSM, Comput. Phys. Commun. 176, 426 (2007).

[15] G. Bélanger, A. Mjallal, and A. Pukhov, Recasting direct detection limits within micrOMEGAs and implication for non-standard dark matter scenarios, Eur. Phys. J. C 81, 239 (2021).

[16] M. Carena, D. Garcia, U. Nierste, and C. E. M. Wagner, Effective lagrangian for the $\bar{t} b H^{+}$interaction in the MSSM and charged Higgs phenomenology, Nucl. Phys. B577, 88 (2000).

[17] S. Marchetti, S. Mertens, U. Nierste, and D. Stockinger, Tan (beta)-enhanced supersymmetric corrections to the anomalous magnetic moment of the muon, Phys. Rev. D 79, 013010 (2009).

[18] G. Degrassi and G. F. Giudice, QED logarithms in the electroweak corrections to the muon anomalous magnetic moment, Phys. Rev. D 58, 053007 (1998).
[19] K. Inoue, M. Kawasaki, M. Yamaguchi, and T. Yanagida, Vanishing squark and slepton masses in a class of supergravity models, Phys. Rev. D 45, 328 (1992).

[20] D. E. Kaplan, G. D. Kribs, and M. Schmaltz, Supersymmetry breaking through transparent extra dimensions, Phys. Rev. D 62, 035010 (2000).

[21] Z. Chacko, M. A. Luty, A. E. Nelson, and E. Ponton, Gaugino mediated supersymmetry breaking, J. High Energy Phys. 01 (2000) 003.

[22] G. Aad et al. (ATLAS Collaboration), Search for electroweak production of charginos and sleptons decaying into final states with two leptons and missing transverse momentum in $\sqrt{s}=13 \mathrm{TeV} p p$ collisions using the ATLAS detector, Eur. Phys. J. C 80, 123 (2020).

[23] A. M. Sirunyan et al. (CMS Collaboration), Search for electroweak production of charginos and neutralinos in proton-proton collisions at sqrt(s) $=13 \mathrm{TeV}$, Technical Report No. CMS-PAS-SUS-19-012, CERN, Geneva, 2021.

[24] E. Aprile et al. (XENON Collaboration), Dark Matter Search Results from a One Ton-Year Exposure of XENON1T, Phys. Rev. Lett. 121, 111302 (2018).

[25] Combined LEP Selectron/Smuon/Stau Results (2004).

[26] J. Hisano and S. Sugiyama, Charge-breaking constraints on left-right mixing of stau's, Phys. Lett. B 696, 92 (2011); Erratum, Phys. Lett. B 719, 472 (2013).

[27] G. H. Duan, C. Han, B. Peng, L. Wu, and J. M. Yang, Vacuum stability in stau-neutralino coannihilation in MSSM, Phys. Lett. B 788, 475 (2019).

[28] D. S. Akerib et al. (LUX-ZEPLIN Collaboration), Projected WIMP sensitivity of the LUX-ZEPLIN dark matter experiment, Phys. Rev. D 101, 052002 (2020).

[29] A. Acharyya et al. (CTA Collaboration), Sensitivity of the Cherenkov telescope array to a dark matter signal from the galactic centre, J. Cosmol. Astropart. Phys. 01 (2021) 057.

[30] P. Cox, C. Han, T. T. Yanagida, and N. Yokozaki, Gaugino mediation scenarios for muon $g-2$ and dark matter, J. High Energy Phys. 08 (2019) 097.

[31] M. Yamaguchi and W. Yin, A novel approach to finely tuned supersymmetric standard models: The case of the nonuniversal Higgs mass model, Prog. Theor. Exp. Phys. (2018), 023B06.

[32] W. Yin and N. Yokozaki, Splitting mass spectra and muon $g$ -2 in Higgs-anomaly mediation, Phys. Lett. B 762, 72 (2016).

[33] T. T. Yanagida, W. Yin, and N. Yokozaki, Flavor-safe light squarks in Higgs-anomaly mediation, J. High Energy Phys. 04 (2018) 012.

[34] G. Aad et al. (ATLAS Collaboration), Searches for electroweak production of supersymmetric particles with compressed mass spectra in $\sqrt{s}=13 \mathrm{TeV} p p$ collisions with the ATLAS detector, Phys. Rev. D 101, 052005 (2020).

[35] R. Franceschini et al., The CLIC potential for new physics, CERN Yellow Rep. Monogr. 3, 101 (2018).

[36] S. Baum, P. Sandick, and P. Stengel, Hunting for scalar lepton partners at future electron colliders, Phys. Rev. D 102, 015026 (2020).

[37] M. Ibe, A. Kamada, and S. Matsumoto, Mixed (cold + warm) dark matter in the bino-wino coannihilation scenario, Phys. Rev. D 89, 123506 (2014). 
[38] K. Harigaya, K. Kaneta, and S. Matsumoto, Gaugino coannihilations, Phys. Rev. D 89, 115021 (2014).

[39] A. M. Sirunyan et al. (CMS Collaboration), Search for physics beyond the standard model in final states with two or three soft leptons and missing transverse momentum in proton-proton collisions at $13 \mathrm{TeV}$, Technical Report No. CMS-PAS-SUS-18-004, CERN, Geneva, 2021.

[40] G. H. Duan, K.-I. Hikasa, J. Ren, L. Wu, and J. M. Yang, Probing bino-wino coannihilation dark matter below the neutrino floor at the LHC, Phys. Rev. D 98, 015010 (2018).
[41] A. M. Sirunyan et al. (CMS Collaboration), Search for supersymmetry in final states with two oppositely charged same-flavor leptons and missing transverse momentum in proton-proton collisions at $\sqrt{s}=13 \mathrm{TeV}$, J. High Energy Phys. 04 (2021) 123.

[42] M. Endo, K. Hamaguchi, T. Kitahara, and T. Yoshinaga, Probing Bino contribution to muon $g-2$, J. High Energy Phys. 11 (2013) 013. 